\title{
A New Privacy-Ware Public Auditing Scheme for Cloud Data Sharing with Group Users
}

\author{
$\mathrm{Mr}^{1}$ V.Jaikumar B.Tech, Mr. ${ }^{2}$ K.Sathish M.E, \\ ${ }^{1}$ Pursuing M.E CSE Dept, ${ }^{2}$ Assistant professor Dept of Computer science and Engineering, \\ Gojan School of Business and Technology, Redhills, Chennai.
}

\begin{abstract}
Cloud computing has been envision as the cutting edge engineering of IT Enterprise. It moves the application programming and information bases to the brought together huge server farms. A conveyed stockpiling system, containing a grouping of limit laborers, gives long stretch amassing organizations over the Internet. Putting away information in an outsider's cloud framework causes genuine worry over information mystery specifically, to accomplish effective information elements, we improve the current confirmation of capacity models by controlling square label verification. To help proficient treatment of different reviewing undertakings, we further investigate the method of mark to expand our primary outcome into a multi-client setting, where TPA can play out numerous evaluating errands all the while. Broad security and execution examination show that the proposed plans are profoundly proficient and provably secure.
\end{abstract}

Keywords - Cloud Computing, Homomorphic Authenticators, Third Party Authority (TPA), Public Auditability Key

\section{INTRODUCTION}

The information stockpiling and sharing administrations given by the cloud, individuals can without much of a stretch work all together by imparting information to one another. All the more explicitly, when a client makes shared information in the cloud, each client in the gathering can get to and change shared information, yet additionally share the most recent rendition of the common information with the remainder of the gathering. Despite the fact that cloud suppliers guarantee a safer and dependable climate to the clients, the uprightness of information in the cloud may in any case be undermined, because of the presence of equipment/programming disappointments and human mistakes In these instruments, a mark is joined to each obstruct in information, and the respectability of information depends on the accuracy of the multitude of marks. Perhaps the most critical and basic highlights of these components is to permit a public verifier to productively check information uprightness in the cloud without downloading the whole information, alluded to as open examining might want to use cloud information for specific purposes (e.g., search, calculation, information mining, and so on) or a Third gathering authority (TPA) who can give confirmation administrations on information respectability to clients. The vast majority of the past works centre around examining the uprightness of individual information. Not the same as these works, a few on-going works centre on how to protect personality security from public verifiers while examining the honesty of shared information. Sadly, nothing unless there are other options instruments considers the effectiveness of client repudiation while evaluating the rightness of shared information in the cloud.

With shared information, when a client adjusts a square, she likewise needs to register another mark for the changed square. Because of the alterations from various clients, various squares are endorsed by various clients. For security reasons, when a client leaves the gathering or makes trouble, this client should be denied from the gathering. Thus, this repudiated client should presently don't have the option to get to and adjust shared information, and the marks created by this disavowed client are not, at this point legitimate to the gathering. Subsequently, albeit the substance of shared information isn't changed during client denial, the squares, which were recently endorsed by the repudiated client, actually should be re-endorsed by a current client in the gathering. Accordingly, the trustworthiness of the whole information can in any case be confirmed with the public keys of existing clients as it were.

\section{Proposed System}

Here we are giving better security in proprietor's transfer side just as on the download side. For better security customer parting that solitary record into nine distinct squares and giving a one of a kind ID number to each 
block. Using Honor Algorithm we are changing over a square tag into secret worth utilizing ASCII value. Client: A substance, which has huge information documents to be put away in the distributed computing and depends on the distributed computing for information upkeep and calculation, During login for a customer here an OTP was produced and that was shipped off the enrolled mail id utilizing that OTP just a customer can login. Third Party Authority (TPA): We are utilizing a public inspecting in proposed an element, which has skill and capacities that customers don't have, is Third to evaluate and uncover danger of distributed computing stockpiling administrations in the interest of the customers upon request. With our instrument, the personality of the underwriter on each square in shared information is kept hidden from public verifiers, who can effectively confirm shared information trustworthiness without recovering the whole document. Furthermore, our instrument can play out various examining errands all the while as opposed to confirming them individually. Our trial results

Exhibit the viability and productivity of our instrument when evaluating shared information honesty. We inspire the public evaluating arrangement of information stockpiling security in Cloud processing, and dynamic information tasks, particularly to help block addition, which is missing in most existing plans. We stretch out our plan to help adaptable and productive public examining in Cloud processing. Specifically, our plan accomplishes reviewing errands from various clients can be performed at the same time by the TPA.Spitted into squares and it is transferred in the distributed computing for better security. We demonstrate the security of our proposed development and legitimize the exhibition of our plan through solid execution and correlations.

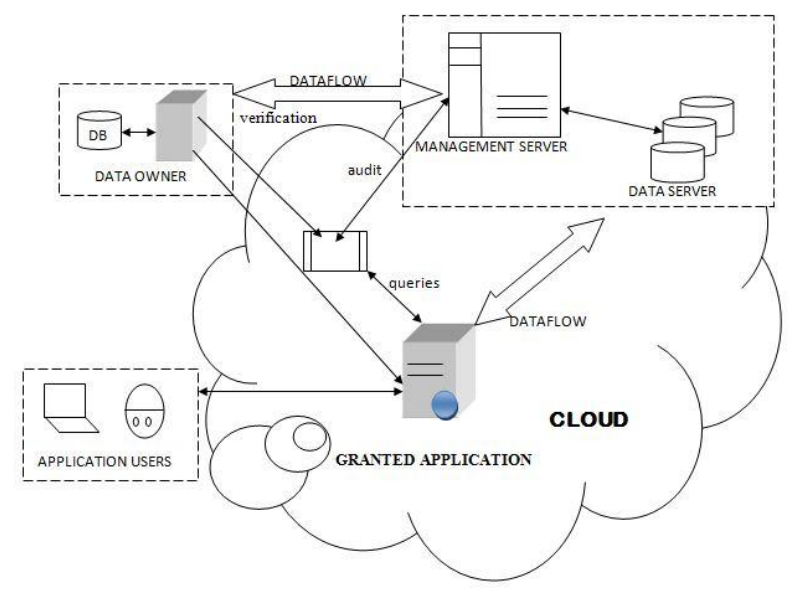

FIG:1 PROPOSED ARCHITECTURE

ADVANTAGE OF PROPOSED SYSTEM:

- We inspire the public examining arrangement of information stockpiling security in Cloud Computing, and propose a convention supporting for completely unique information tasks, particularly to help block inclusion, which is missing in most existing plans.

- We stretch out our plan to help versatile and productive public examining in Cloud Computing. Specifically, our plan accomplishes reviewing assignments from various clients can be performed at the same time by the TPA.

- We demonstrate the security of our proposed development and legitimize the exhibition of our plan through solid execution and correlations

\section{Problem statement}

\subsection{SYSTEM MODEL:}

This application includes three gatherings: the cloud worker, the outsider evaluator (TPA) and clients. There are two sorts of clients in a gathering: the first client and various gathering clients. Third Party Auditing 


\section{MODULES}

- User Interface

- $\quad$ Mail Alert Process

- File Retrieval And Error Recovery And Batch Auditing For Multi-Client Data

- Verification Phase

\section{USER INTERFACE:}

Users can use them from wherever at any time. For instance, the email administration is presumably the most well-known one. Distributed computing is an idea that treats the assets on the Internet as a bound together element, a cloud. Clients simply use administrations without being worried about how calculation is done and capacity is overseen. In this paper, we centre on planning a distributed storage framework for heartiness, privacy, and usefulness. A cloud storage system is careful user interface entry level formation in this module.

\section{MAIL ALERT PROCESS:}

The uploading and downloading process of the user is first get the secret key in the corresponding user email id and then apply the secret key to encrypted data to send the server storage and decrypts it by using his secret key to download the corresponding data file in the server storage system's the secret key conversion using the Share Key Gen (SKA, t, m). This algorithm shares the secret key SKA of a user to a set of key servers.

\section{FILE RETRIEVAL AND ERROR RECOVERY AND BATCH AUDITING FOR MULTI-CLIENT DATA:}

Since our layout of file matrix is systematic, the user can reconstruct the original file by downloading the data vectors from the first $\mathrm{m}$ servers, assuming that they return the correct response values. Notice that our verification scheme is based on random spot-checking, so the storage correctness assurance is a probabilistic one. We can guarantee the successful file retrieval with high probability. On the other hand, whenever the data corruption is detected, the comparison of pre-computed tokens and received response values can guarantee the identification of misbehaving server(s). As cloud servers may concurrently handle multiple verification sessions from different clients, given $\mathrm{K}$ signatures on $\mathrm{K}$ distinct data files from $\mathrm{K}$ clients, it is more advantageous to aggregate all these signatures into a single short one and verify it at one time. To achieve this goal, we extend our scheme to allow for provable data updates and verification in a multi-client system. The mark conspire permits the production of marks on subjective particular messages. Moreover, it supports the aggregation of multiple signatures by distinct signers on distinct messages into a single short signature, and thus greatly reduces the communication cost while providing efficient verification for the authenticity of all messages.

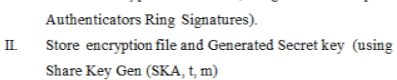

Store encryption file and Generated Secret key (using Share Key Gen (SKA, t, m)

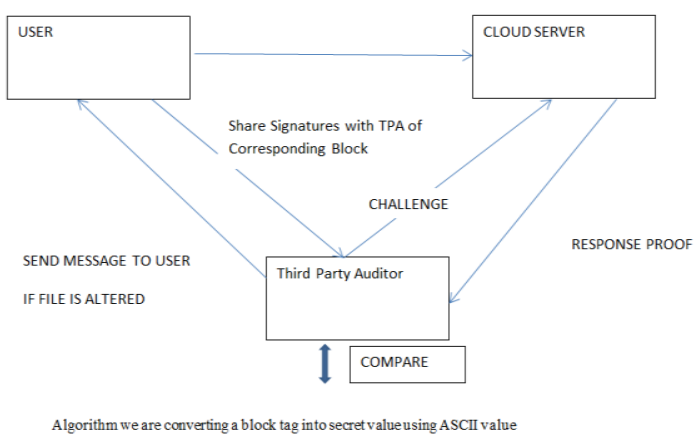

FIG: 2 DATA PROCESSING USING TPA 


\section{VERIFICATION PHASE:}

The verifier prior to putting away the record at the file pre-measures the document and attaches some Meta information to the record and stores at the chronicle. At the hour of check the verifier utilizes this Meta information to confirm the honesty of the information. Note that our evidence of information trustworthiness convention simply checks the uprightness of information i.e. if the data has been illegally modified or deleted. It does not prevent the archive from modifying the data. Now we show how our scheme can explicitly and efficiently handle fully dynamic data operations including data modification (M), data insertion (I) and data deletion (D) for cloud data storage. Note that in the following descriptions, we assume that the file $\mathrm{F}$ and the signature have already been generated and properly stored at server. The root metadata $\mathrm{R}$ has been signed by the client and stored at the cloud server, so that anyone who has the client's public key can challenge the correctness of data storage.

\section{THIRD PARTY AUDITING:}

As discussed in our architecture, in case the user does not have the time, feasibility or resources to perform the storage correctness verification, he can optionally delegate this task to an independent Third party authority, making the cloud storage publicly verifiable. However, as pointed out by the recent work, to securely introduce an effective TPA, the auditing process should bring in no new vulnerabilities towards user data privacy. Namely, TPA should not learn user's data content through the delegated data auditing.

\section{$\underline{\text { Admin Service Providers }}$}

(1) Update Operation:

In cloud data storage, sometimes the user may need to modify some data block(s) stored in the cloud, we refer this operation as data update. In other words, for all the unused tokens, the user needs to exclude every occurrence of the old data block and replace it with the new one.

\section{(2) Delete Operation:}

Sometimes, after being stored in the cloud, certain data blocks may need to be deleted. The delete operation we are considering is a general one, in which user replaces the data block with zero or some special reserved data symbol. From this point of view, the delete operation is actually a special case of the data update operation, where the original data blocks can be replaced with zeros or some predetermined special blocks.

\section{(3) Append Operation:}

In some cases, the user may want to increase the size of his stored data by adding blocks at the end of the data file, which we refer as data append. We anticipate that the most frequent append operation in cloud data storage is bulk append, in which the user needs to upload a large number of blocks (not a single block) at one time.

\section{IV.TECHNIQUES AND ALGORITHM USED:}

The client can likewise encode information prior to rethinking it into the cloud worker with encryption procedures .As a huge examination region for framework insurance, information access control has been advancing in the previous thirty years and different strategies have been created to viably carry out fine-grained admittance control, which permits adaptability in indicating differential access privileges of individual clients. Conventional access control models typically expect the information proprietor and the workers putting away the information are in a similar Third space, where the workers are completely an unthird as an all-knowing reference screen answerable for characterizing and upholding access control approaches. This suspicion anyway no longer holds in distributed computing since the information proprietor and cloud workers are probably going to be in two distinct spaces. With the personality of low support, distributed computing gives a conservative and productive answer for sharing gathering asset among cloud clients. Tragically, sharing information in a multiproprietor way while protecting information and personality security from an un-third cloud is as yet a difficult issue, because of the regular difference in the enrollment. In this paper, we propose a protected multi-proprietor information sharing plan, named Mona, for dynamic gatherings in the cloud. By utilizing bunch signature and dynamic transmission encryption procedures, any cloud client can secretly impart information to other people. In the meantime, the capacity overhead and encryption calculation cost of our plan are autonomous with the quantity of renounced clients. Moreover, we investigate the security of our plan with thorough verification, and exhibit the productivity of our plan in tests. 


\subsection{IMPLEMENTATION OF ALGORITHM:}

- Homomorphic Authenticators Ring Signatures

- $\quad$ Rijndael Managed, Suppose that a group of entities each have public/private key pairs, (PK1, SK1), (PK2, SK2), ..., (PKn, SKn).

- Gathering I can figure a ring mark $\sigma$ on a message $\mathrm{m}$, on input (m, SKi, PK1, ...,PKn). Anybody can check the legitimacy of a ring mark given $\sigma, \mathrm{m}$, and the public keys included, PK1, ..., PKn. In the event that a ring mark is appropriately registered, it should pass the check.

- On the other hand, it should be hard for anyone to create a valid ring signature on any message for any group without knowing any of the secret keys for that group

- $\quad$ Ring marks, rst presented by Rivest, Shamir, and Tauman, empower a client to sign a message so a ring of plausible underwriters (of which the client is a part) is recognized, without uncovering precisely which individual from that ring really created the mark.

- As opposed to amass marks, ring marks are totally impromptu" and don't need any focal position or coordination among the different clients (undoubtedly, clients don't should know about one another); moreover, ring mark plans award clients ne-grained authority over the degree of obscurity related with a specific mark. This paper has two fundamental regions of core interest.

- In the first place, we inspect past meanings of safety for ring mark plots and recommend that the majority of these earlier definitions are too powerless, as in they don't consider certain practical assaults.

- We propose new definitions anonymity and unforgetability which address these threats, and give separation results proving that our new notions are strictly stronger than previous ones second, we show the rst developments of ring mark plans in the standard model.

- One plan depends on conventional suppositions and fulfils our most grounded meanings of safety.

- Two additional schemes are more recent, but achieve weaker security guarantees and more limited functionality.

\subsection{OBSERVATIONS:}

The perceptions of our proposed structure provoked the occurrence of the results referred to under:

\section{Mixed data and key are taken care of autonomously on different limit media.}

2. Before disentangling the data the customer need to enter OTP which is sent on his mail and mix of OTP, key and mixed data are used to deliver remarkable data.

3. For getting to the data the customer is restricted in read just mode and for insert, change and eradicate the notification is dispatched off manager.

4. After encryption or unscrambling the primary data is eradicated.

5. For getting the Account and Service Hijacking, we are murdering the TPA. Created by TPA will be done by head and our proposed system.

\section{Pre-Process and Post Process Work}

\section{A.Ring Signatures:}

The idea of ring marks is first proposed by Rivest et al. With ring marks, a verifier is persuaded that a mark is registered utilizing one of gathering individuals' private keys; however the verifier can't figure out which one. This property can be utilized to protect the personality of the endorser from a verifier. The ring mark plot presented by Boneh et al. We will stretch out this ring mark plan to build our public reviewing instrument. 


\section{B.Homomorphic Authenticators SIGN:}

Homomorphic authenticators (likewise called homomorphic evident labels) are essential devices to build information reviewing instruments. Other than Enforceability (just a client with a private key can produce legitimate marks), a homomorphic authenticable mark conspires, which means a homomorphic authenticator dependent on marks, ought to likewise fulfil the accompanying properties:

\section{C.Blockless Verification:}

Blockless affirmation allows a verifier to audit the precision of data set aside in the cloud specialist with a single square, which is an immediate blend of the overall large number of squares in data. In case the combined square is correct, the verifier acknowledges that the squares in data are for the most part right. Thusly, the verifier doesn't need to download all of the squares to check the genuineness of data.

\section{D.Non-Malleability:}

Non-flexibility demonstrates that an assailant can't produce substantial marks on invalid squares by directly joining existing marks.

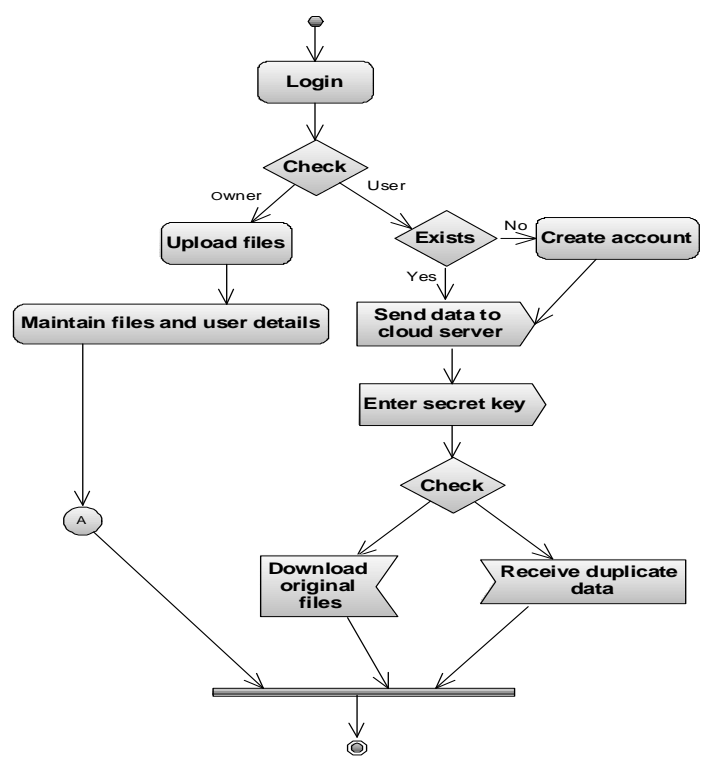

FIG: 3 DEVELOPMENT OF PROJECT CREATION IN TPA

\section{E. Performance Metrics:}

Applications like information investigation, insights for the most part includes in preparing enormous measure of information. Each datasets relates to assortment of information, factors or separate data set credits. In such circumstance it is fundamental to investigate the exhibition of the different ascribes. 


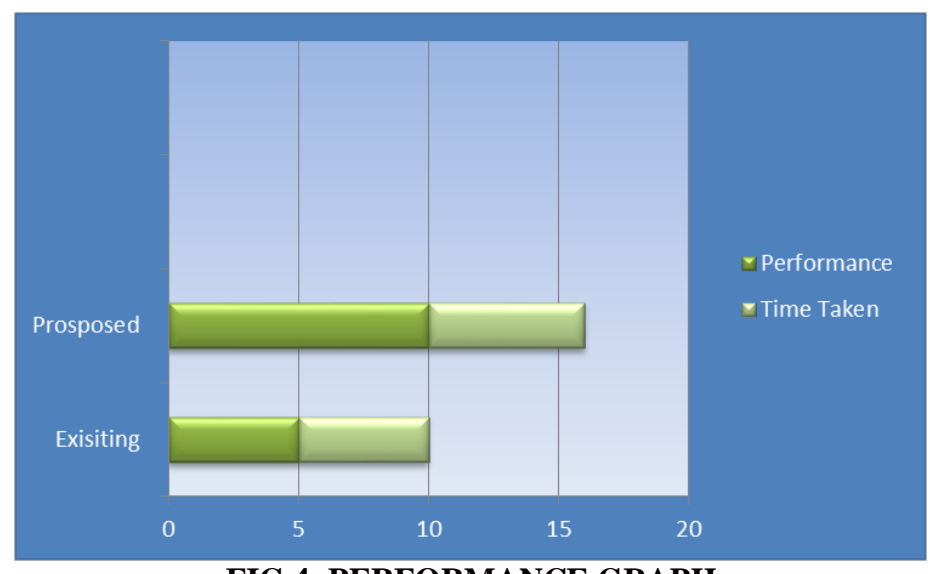

FIG 4: PERFORMANCE GRAPH

As shown in the graph considering the performance against the time we could see that the proposed system Grows better in its performance with respect to the efficiency for recommending the relevant information the User interested. It is very important to recommend the most popular places to user which is also interested and liked by the same user.

\section{Conclusion}

We propose a protection saving public examining framework for information stockpiling security in Cloud Computing. We use the homomorphic authenticator and irregular concealing to ensure that TPA would not become familiar with any information about the information content put away on the cloud worker during the proficient evaluating measure, which not just kills the weight of cloud client from the drawn-out and conceivably costly reviewing task, yet in addition reduces the clients' dread of their rethought information spillage. Considering TPA may simultaneously deal with various review meetings from various clients for their rethought information records, we further broaden our protection safeguarding public inspecting convention into a multi-client setting, where TPA can play out the different evaluating errands in a group way.

\subsection{FUTURE ENHANCEMENT:}

We propose a security safeguarding public reviewing component for shared information in the cloud permits a verifier to check the rightness of a customer's information put away at an unThird worker. By using RSA-based homomorphic authenticators and inspecting methodologies, the verifier can freely review the honesty of information without recovering the whole information, which is alluded to as open examining. Lamentably, their system is just appropriate for inspecting the respectability of individual information. Comparable model called Proofs of Irretrievability (POR), which is likewise ready to check the rightness of information on an unThird worker. The first record is added with a bunch of haphazardly esteemed check blocks called sentinels. The verifier challenges the unThird worker by determining the places of an assortment of sentinels and requesting that the unThird worker return the related sentinel esteems. Shacham and Waters planned two improved plans. The principal conspire is worked from BLS marks, and the subsequent one depends on pseudo-irregular capacities. We use ring marks to build homomorphic authenticators, so a public verifier can review shared information respectability without recovering the whole information, yet it can't recognize who is the underwriter on each square.

\section{REFERENCES}

[1] Chelvan, A. Chilambu, and M. Rajesh. "Optimization of Secret Key using cuckoo Search Algorithm for ensuring data integrity in TPA." 2020 International Conference on Computer Communication and Informatics (ICCCI). IEEE, 2020.

[2] Ouyang, Hongbing, Xiaolu Wei, and Qiufeng Wu. "Discovery and Prediction of Stock Index Pattern via Three-Stage Architecture of TICC, TPA-LSTM and Multivariate LSTM-FCNs." IEEE Access 8 (2020): 123683-123700

[3] Chen, Jiehong, Shuhua Qi, and JunsongJia. "The Comparisons between TPA and Cloude-Pottier Alpha Angle." 2019 SAR in Big Data Era (BIGSARDATA). IEEE, 2019.

[4] Ismail, RashaRokan, and Taha Mohammed Hasan. "Improving Security and Sharing Management in Cloud Computing Using TPA." 2019 1st AL-Noor International Conference for Science and Technology (NICST). IEEE, 2019.

[5] Apritama, Muhammad Rizki, et al. "Removal of BOD and COD in TPA Leachate Using Subsurface Constructed Wetland with Equisetum hyemale." 2019 2nd International Conference on Applied Engineering (ICAE). IEEE, 2019. 